\title{
Multiobjective Model for Emergency Resources Allocation
}

\author{
Zhaosheng Yang, ${ }^{1,2}$ Huxing Zhou, ${ }^{1}$ Xueying Gao, ${ }^{1}$ and Songnan Liu ${ }^{1}$ \\ ${ }^{1}$ College of Traffic, Jilin University, Changchun 130012, China \\ ${ }^{2}$ State Key Laboratory of Automobile Dynamic Simulation, Jilin University, Changchun 130012, China
}

Correspondence should be addressed to Huxing Zhou; zhouhuxing@gmail.com

Received 7 August 2012; Revised 21 November 2012; Accepted 18 December 2012

Academic Editor: Wuhong Wang

Copyright (C) 2013 Zhaosheng Yang et al. This is an open access article distributed under the Creative Commons Attribution License, which permits unrestricted use, distribution, and reproduction in any medium, provided the original work is properly cited.

\begin{abstract}
Emergency resources allocation is essential to the emergency rescue effectiveness, and it has become a research focus for emergency rescue. This paper proposes a multiresource dynamic allocation model of emergency rescues and corresponding solving method. The object of the proposed model is to maximize the overall emergency rescue effectiveness of allocated resources and minimize the allocating costs of resources. The model considers the dynamic nature that the casualties of trapped victims change over time. At last, a numerical example is presented to test the model and its algorithm.
\end{abstract}

\section{Introduction}

Emergency resources allocation is the material foundation of the emergency rescue supplies and can greatly improve the supply efficiency of the emergency resources. In this paper, the emergency resources allocation studies primarily aim at facing the multihazard disaster threat during the emergency regional preparedness. A multistage resources allocation optimization model is formulated which is more dynamic based on the temporal and spatial variation features of the emergency demand.

Alsalloum and Rand studied the minimum quantity and the location optimization of the emergency relief ambulance, showing how to choose the right location for the ambulance to maximize the expected demand coverage while minimizing the number of vehicles with the goal programming method. Rajagopalan et al. proposed a model to meet the dynamic demands of the ambulance during a certain period of time $[1,2]$. Yi and Özdamar came up with an integrated location distribution model for coordinating logistics support and evacuation operations in disaster response activities, which is an LRP problem [3]. Chang et al. formulated the flood emergency logistics problem with uncertainty as two stochastic programming models, the grouping and classifying model and the location-allocation model, which allow the determination of a rescue resource distribution system for urban flood disasters [4]. Rajagopalan et al. formulated the dynamic available coverage location (DACL) model to determine the minimum number of ambulances and their locations for each time cluster in which significant changes in demand pattern occur while meeting coverage requirements with a predetermined reliability and used the tabu search algorithm to obtain its globally optimal solutions [2]. In order to strengthen natural disaster prevention mechanism, Rawls and Turnquist developed an emergency response planning tool to obtain the emergency preparedness resources locations and inventory levels under uncertain situation [5]. Mete and Zabinsky proposed a stochastic optimization approach for the storage and distribution problem of medical supplies to be used for disaster management under a wide variety of possible disaster types and magnitudes [6].

\section{Multiresource Dynamic Allocation Model for Emergency Rescue}

The rationality of rescue resource allocation directly affects the effectiveness of rescue. Strategic decision of rescue resource allocation is influenced by the variety, scale, cost, and various factors. Overladen resource reserve would cause massive waste of money and resource, while too little reserve would seriously reduce rescue effectiveness.

2.1. Problem Description. The rescue resource allocation discussed in this paper is about dealing with the allocation for 
frequent disasters in regular resource spot. Since the threat come from disasters, the rescue demand and resources characteristics are dynamically changeable, the rescue resource allocation should also be dynamically changeable along with the situation of the disaster. Furthermore, given that frequent disasters happen with strong regular patterns, the rescue demands in different periods of disaster can be estimated according to historic disaster records; thus, the rescue resource could also be rationally allocated. Scientific rescue resource allocation should be based on the following considerations.

(1) The importance of resource. The importance of rescue resource refers to the utility of resource in rescue [7]. It is mainly reflected in the replacement of resource and the effect on rescue. Hereinafter, the fungible resources are seen as the same to simplify the problem without affecting resource allocation. So the extent that resource has effect on disasters is used to reflect the importance of resource. To reflect the importance of various types of resources more directly, we could hereby quantify the extent of effect on disasters brought by every types of resources, and then the importance of resources could be represented by a range of figures, for example 1-10 grades.

(2) The timeliness of resource. The rescue resource is valuable only when it is delivered to the demanders [8]. The amount of survivors reduces with the loss of time, and the quantity of resources which could have effect in rescue also decreases. When the arrival of rescue resource has exceeded the time limit of rescue resource, the arrival of resources is meaningless.

(3) The scarcity degree of resource. Resource scarcity degree is the degree of difficulty of collect or purchase. Rare resources, especially in emergent need, should be stored; otherwise, when needed, collecting the necessary resources would take too much time to miss the best rescue time and cause serious casualties. For the resources which could be easily purchased and collected, the storage should be rationally reduced to save storage cost without causing bad effects on rescue.

(4) The cost of resource. The purpose of rescue resource storage is for emergency, but the cost should not be ignored. The scale of rescue resource is usually massive, and the investment from the government is also numerous. By allocating the scale of resource storage reasonably to reduce the cost of purchasing and storage would highly ease the fiscal tension of local rescue department.

2.2. Model Formulation. For easily describing and understanding the model, the model construction should satisfy the 3 premise assumptions as the following.

(1) Because of the strong discipline of frequent disasters, we assume that the type, range, and degree of possible disasters and the expected value of types and quantity of resources in different periods from different demanding spots are expectable.

(2) Resource spots and demanding spots are all connected, with time of arrival known between every two spots.

(3) Each resource spot delivers each type of resource to every demanding spot at most.

Multiresource dynamic allocation model for emergency rescues is a multigoal optimization model with two goals, maximizing rescue effectiveness and minimizing allocating cost.

(1) Maximum of rescue effectiveness. The purpose of rescue resource allocation is to deal with emergencies, so the maximum of rescue effectiveness is the first goal and the maximum of the importance of all the resources could reflect this goal. On the other hand, according to the dynamic change of the threat of the disasters, the rescue period could be divided into several time intervals, and the model seeks the maximum of rescue resource effectiveness during the whole projection period. The so-called "maximum of rescue resource effectiveness" here concerns not only the effectiveness of the resource allocation, but also the maximum of the whole rescue project with the consideration of the whole rescue demands.

The effectiveness of rescue resource has important relationship with the scarcity degree of resource. The timeliness of resource is reflected by the amount of resources which is still of value with the loss of time and also matters the casualties of the victims. The scarcity degree of resource could be seen as the length of time needed for preparation. The resource with higher scarcity need longer time to be transfered and be produced from other place. So the time for preparation could be used to represent the degree of scarcity of resource. The so-called "time for preparation" means the time needed for the arrival of rescue resource at the demanding spot after disaster happens. The first goal of the model is as the following:

$$
\begin{aligned}
\text { Obj } 1 \quad f_{1}=\max \sum_{k=1}^{o} \sum_{l=1}^{p} & \left(u_{l} \sum_{i=1}^{n} \sum_{j=1}^{m} x_{i j k l} f_{j k l}\left(t_{i j}\right)\right. \\
& \left.+u_{l}\left(d_{j k l}-\sum_{i=1}^{n} \sum_{j=1}^{m} x_{i j k l}\right) f_{j k l}\left(t_{\text {rep } j}\right)\right) .
\end{aligned}
$$

In (1), $i$ represents the number of resource spot; $j$ represents the number of demand resource spot; $k$ represents the number of response time; $l$ represents the number of resources type; $u_{l}$ represents the utility value of $l$ type resources per unit; $x_{i j k l}$ is the amount of $l$ type resources delivered from point $i$ to point $j$ during the time period $k ; d_{j k l}$ represents the demand resources of $l$ type that point $j$ needed during the time period $k ; t_{i j}$ is the transfer time from point $i$ to 
TABLE 1: Conditions of the threat of disasters that each region faced at all stages.

\begin{tabular}{lccccc}
\hline \multicolumn{1}{c}{$\begin{array}{c}\text { Analytical stages } \\
\text { Types of disasters }\end{array}$} & I & \multicolumn{2}{c}{ II } & III \\
\hline Demand spots & 1 & 0.7 & 0.5 & 0.7 & 0.4 \\
Disasters & 2 & 0.5 & 0.8 & 0.3 & 0.8 \\
Occurrence & 3 & 0.2 & 0.7 & 0.8 & 0.6 \\
Probability & 4 & 0.8 & 0.2 & 0.2 & 0.6 \\
\hline
\end{tabular}

point $j ; t_{\text {rep } j}$ is the preparation time of $j$ type rescue resources; the rescue time-effect function of the $l$ type resources needed by demand spot $j$ at moment $t$ in period $k$ is given by $f_{j k l}(t)$.

Through (1), we can find that the section before the plus sign is the emergency rescue utility value of allocated resources, while the section after the plus sign is the emergency rescue utility value of unallocated resources which need temporary preparation. This objective can reflect the total effectiveness of the whole emergency resources allocation proposal in the planning period.

(2) The lowest-cost allocation of resources. The cost of emergency response resources are divided into three parts: purchasing cost, inventory cost, and opportunity loss cost, $c_{a l}$ and $c_{b l}$, respectively, represent purchase cost and inventory cost. Opportunity loss cost is due to a shortage of resources in disasters, which results in that resources which purchase cost will be higher than usual. Temporarily purchasing the unreserved emergency resources will cause a certain loss. Consequently, it will increase the cost of the emergency rescue work, which is the focus of this study. We use $o_{p i}$ to represent the unit opportunity loss cost of the $l$ type resources. Besides, most resource spots have a certain economic functions, so if a certain disaster did not happen that the corresponding resources can be sold to reduce the cost of the resources allocation. Assume that the sale of a resource per unit will earn $p_{r l}$ profit and the possibility of the $q$ type disaster happening on demand spot $j$ during period $k$ is $\alpha_{j k q}$. We use $N_{j k 1}$ to represent the demand of the $l$ type resources of the point $j$ during the period $k$. The objective of the lowest total cost of the allocation resources is given as the following:

$$
\begin{aligned}
\text { Obj2 } f_{2}=\min \sum_{j=1}^{m} \sum_{k=1}^{o} \sum_{l=1}^{p}\left(\sum_{i=1}^{n}(\right. & \left(c_{a l}+c_{b l}\right) \cdot x_{i j k l} \\
& \left.\quad-p_{r l} \cdot \sum_{q=1}^{v}\left(x_{i j k l} \cdot\left(1-\alpha_{j k q}\right)\right)\right) \\
& \left.+o_{p l} \cdot\left(N_{j k l}-\sum_{i=1}^{n} x_{i j k l}\right)\right) .
\end{aligned}
$$

The temporary emergency resources location model formulated should satisfy the following constraints.

(1) The total amount of available stored resources at different stages allocated cannot exceed its reserve capacity. Here is the mathematical expression of the following constraint equation (3), $N_{i k}$ is the reserve capacity of the resource spot $i$ :

$$
\sum_{j=1}^{m} \sum_{l=1}^{p} x_{i j k l} \leq N_{i}
$$

(2) The total resources that supply all kinds of resources spot cannot exceed the actual total demand. It is given by (4):

$$
\sum_{i=1}^{n} x_{i j k 1} \leq N_{j k 1} .
$$

(3) The amount of the resources allocated to each resources spot should be a positive number, as shown in (5):

$$
x_{i j k 1} \in\left\{0, R^{+}\right\} \text {. }
$$

The proposed dynamic emergency rescues allocation model is a typical multiobjective programming model. Similar to above-mentioned optimization location model, the requirements of both objectives should be considered in an integrative way. The model is solved by the ideal point method (IPM) in order to achieve a noninferior solution with the result of each subobjective close to the optimum value. The result derived from this method can be further improved by utilizing the idea of the unit cost utility method, as demonstrated in (6):

$$
\mathrm{Obj}^{* *} \quad \max \frac{f_{1}}{f_{2}} \text {. }
$$

The details of the solving steps are as follows.

Step 1. Solve the model composed of (1) and (3)-(5) for an optimized value (here we go for the minimum value with a negated object), denoted as $f_{1}^{*}$, go to Step 2 .

Step 2. Solve the model composed of (2)-(5) except (1) for an optimized value, denoted as $f_{2}^{*}$, go to Step 3 .

Step 3. Solve the model composed of (7), (3)-(5) for an optimized value, denoted as $f_{3}^{*}$, go to Step 4 ,

$$
\text { Obj } \min f_{3}=\sqrt{\left(-f_{1}-f_{1}^{*}\right)^{2}+\left(f_{2}-f_{2}^{*}\right)^{2}} \text {. }
$$

Step 4. Construct the objective function and constrains, as illustrated in (8) and (9), (8) is set as the objective for 
TABLE 2: Quantities of the demand of resources that different demand spots respond to various types of disasters.

\begin{tabular}{|c|c|c|c|c|c|c|c|}
\hline \multirow{2}{*}{$\begin{array}{r}\text { Types of disasters } \\
\text { Types of resources }\end{array}$} & & \multicolumn{2}{|c|}{ A } & \multirow{2}{*}{$\begin{array}{l}\mathrm{B} \\
3\end{array}$} & \multicolumn{2}{|c|}{$\mathrm{C}$} & \multirow{2}{*}{$\begin{array}{l}\mathrm{D} \\
6\end{array}$} \\
\hline & & 1 & 2 & & 4 & 5 & \\
\hline Effectiveness of resource & & 5 & 8 & 6 & 5 & 9 & 7 \\
\hline \multirow{4}{*}{$\begin{array}{l}\text { All types of resources required } \\
\text { for demand spots }\left(\mathrm{m}^{3}\right)\end{array}$} & 1 & 1500 & 2000 & 1200 & 1200 & 1200 & 800 \\
\hline & 2 & 1200 & 1500 & 2500 & 500 & 400 & 2400 \\
\hline & 3 & 600 & 800 & 2400 & 2000 & 1800 & 1800 \\
\hline & 4 & 2000 & 2500 & 500 & 300 & 300 & 1800 \\
\hline
\end{tabular}

TABLE 3: Scales of resources spots.

\begin{tabular}{lccc}
\hline Resources spots & 1 & 2 & 3 \\
Scales $\left(\mathrm{m}^{3}\right)$ & 5000 & 5000 & 6000 \\
\hline
\end{tabular}

TABLE 4: Shortest time of transportation between resource and demand spots (h).

\begin{tabular}{lccc}
\hline Demand spots & \multicolumn{3}{c}{ Resources spots } \\
\hline 1 & 1 & 2 & 3 \\
2 & 35 & 25 & 20 \\
3 & 30 & 50 & 28 \\
4 & 45 & 30 & 56 \\
\hline
\end{tabular}

TABLE 5: Time of preparation that each demand spot needs all types of emergency resources $(h)$.

\begin{tabular}{lcccc}
\hline Resources & \multicolumn{4}{c}{ Demand spots } \\
\hline 1 & 1 & 2 & 3 & 4 \\
2 & 35 & 35 & 40 & 40 \\
3 & 48 & 48 & 48 & 48 \\
4 & 30 & 25 & 36 & 30 \\
5 & 40 & 20 & 20 & 50 \\
6 & 30 & 30 & 40 & 50 \\
\hline
\end{tabular}

TABLE 6: Cost associated with all types of resources.

\begin{tabular}{lcccccc}
\hline Resource & 1 & 2 & 3 & 4 & 5 & 6 \\
\hline$c_{a i}\left(¥ / \mathrm{m}^{3}\right)$ & 200 & 120 & 240 & 160 & 160 & 100 \\
$c_{b i}\left(¥ / \mathrm{m}^{3}\right)$ & 10 & 8 & 10 & 8 & 10 & 8 \\
$p_{r l}\left(¥ / \mathrm{m}^{3}\right)$ & 160 & 100 & 200 & 20 & 40 & 80 \\
$o_{p l}\left(¥ / \mathrm{m}^{3}\right)$ & 30 & 0 & 40 & 30 & 40 & 0 \\
\hline
\end{tabular}

optimization and (2)-(5) and (9) are used as constrains, the resulting solution is the optimized solution:

$$
\begin{gathered}
\text { Obj } \max f_{4}=\frac{f_{1}}{f_{2}} \\
f_{1}=f_{3}^{*} .
\end{gathered}
$$

Solutions are carried out by the software LINGO.
TABLE 7: Algorithm solution obtained from ideal point effectiveness of unit cost method $\left(\mathrm{m}^{3}\right)$.

\begin{tabular}{lcccc}
\hline \multirow{2}{*}{ Analytical stages } & Resources & \multicolumn{3}{c}{ Resource spots } \\
& & 1 & 2 & 3 \\
\hline I & 1 & 0 & 600 & 1500 \\
& 2 & 1963 & 800 & 4037 \\
& 3 & 0 & 2181 & 1200 \\
II & 4 & 167 & 0 & 1333 \\
& 5 & 169 & 1800 & 1331 \\
& & & & \\
III & 6 & 681 & 1800 & 4319 \\
\hline
\end{tabular}

TABLE 8: Solutions obtained by ideal points $\left(\mathrm{m}^{3}\right)$.

\begin{tabular}{lcccc}
\hline \multirow{2}{*}{ Analytical stages } & Resources & \multicolumn{3}{c}{ Resource spots } \\
& & 1 & 2 & 3 \\
\hline I & 1 & 2128 & 600 & 2500 \\
& 2 & 2500 & 800 & 3500 \\
& 3 & 0 & 2400 & 1200 \\
II & 4 & 159 & 0 & 1341 \\
& 5 & 158 & 1800 & 1342 \\
& & & & \\
III & 6 & 900 & 1800 & 4100 \\
\hline
\end{tabular}

\section{Experimental Result}

We proposed a numerical example to verify the emergency rescue resource allocation model. Assume that there are four demand spots in the analytical region, and the conditions of the region threatened by the disaster on average per year can be roughly divided into three stages during the planning period. The types of disasters and the quantities and types of required emergency resources in each stage are shown in Tables 1 and 2, respectively. There are three fixed resource spots in the region, and the scale of each resource spot is shown in Table 3. The time of transportation between each resource and demand spot is shown in Table 4, and the preparation time of all types of emergency resources each demand spot needs is show in Table 5. The cost of purchase and maintenance of all kinds of resources and the income of resources sale are shown in Table 6, the total investment of the allocation of resources does not exceed of $¥ 3.5$ million. The functions of emergency timeliness of corresponding 
TABLE 9: Solutions obtained by the maximum effectiveness of single target.

\begin{tabular}{lcccr}
\hline Analytical stages & Resources & \multicolumn{2}{c}{ Resource spots } & \multicolumn{1}{c}{3} \\
\hline I & 1 & 2200 & 600 & 2500 \\
& 2 & 2500 & 800 & 3500 \\
& 3 & 0 & 2400 & 1200 \\
II & 4 & 0 & 0 & 1500 \\
& 5 & 0 & 1800 & 1500 \\
III & 6 & 0 & 1800 & 5000 \\
\hline
\end{tabular}

TABLE 10: Comparison of indicators related to algorithm solution obtained from three methods.

\begin{tabular}{lccc}
\hline Solutions & $\begin{array}{c}\text { Emergency effectiveness } \\
\text { (the first target) }\end{array}$ & $\begin{array}{c}\text { Total cost of configuration } \\
\text { (million } ¥)\end{array}$ & $\begin{array}{c}\text { Cost effectiveness of } \\
\text { configuration }\end{array}$ \\
\hline $\begin{array}{l}\text { Ideal point effectiveness of } \\
\text { unit cost }\end{array}$ & 173719 & 325 & 536 \\
$\begin{array}{l}\text { Deal point } \\
\text { The maximum effectiveness } \\
\text { of single target }\end{array}$ & 173719 & 368 & 472 \\
\hline
\end{tabular}

resources about different demand spots respond to various types of disasters are as the following:

$$
\begin{aligned}
& q_{A}(t)=\exp \left(-0.1\left(\frac{t}{10}\right)\right) \\
& q_{A}(t)=\exp \left(-0.2\left(\frac{t}{24}\right)^{1.5}\right) \\
& q_{B}(t)=\exp \left(-0.3\left(\frac{t}{32^{2}}\right)\right) \\
& q_{C}(t)=\exp \left(-0.8\left(\frac{t}{48}\right)^{2}\right) \\
& q_{D}(t)=\exp \left(-0.1\left(\frac{t}{18^{2}}\right)\right) \\
& q_{D}(t)=\exp \left(-0.2\left(\frac{t}{54}\right)^{2.5}\right) .
\end{aligned}
$$

The optimized solution based on the algorithm is shown in Table 7. The solution of Step 4 without the algorithm above, namely, only use of the ideal point is shown in Table 8. Regardless of cost, that is, remove the second objective to obtain the solution which is shown in Table 9. The comparison of indicators related to algorithm solution obtained from three methods is shown in Table 10.

From Table 10, it can be seen that IPM may take the first and second objectives into account to some extent. The total value of effectiveness of IPM declines compared with the model which only considers the first objective, while the configuration costs are reduced, the effect is not obvious.

The cost and the emergency effectiveness are only reduced by $0.8 \%$ and $0.7 \%$, respectively, but by the proposed algorithm, the cost of resources allocation is reduced by $12.4 \%$ under the condition that the emergency effectiveness only is reduced by $0.7 \%$. The quality of the optimization is greatly improved.

\section{Conclusions}

Based on the actual demand of the emergency rescue resources allocation, this paper proposes multiemergency rescue dynamic allocation model. The model considers the dynamic nature that the casualties of trapped victims change over time. The model for the allocation of rescue resources aims at maximizing the overall emergency rescue effectiveness of rescue proposal of the allocated and the unallocated resources at each stage during the planning period, and it also allows for the cost of allocation resources. Considering the purchasing cost, inventory cost, and opportunity loss cost during the cost objectives construction, we propose the method that combines ideal point method and unit cost utility method to solve the allocation model by utilizing the LINGO software. At the end of this paper, we design the numerical example to test the model and its algorithm.

\section{Conflict of Interests}

The authors claim that there is not any conflict of interests in their research.

\section{Acknowledgment}

Zhaosheng Yang express his sincere appreciation to the Chinese National High Technology Research and Development Program Committee no. 2009AA11Z208 and Project 20121100 supported by Graduate Innovation Fund of Jilin University for the financial support of this paper. 


\section{References}

[1] O. I. Alsalloum and G. K. Rand, "Extensions to emergency vehicle location models," Computers and Operations Research, vol. 33, no. 9, pp. 2725-2743, 2006.

[2] H. K. Rajagopalan, C. Saydam, and J. Xiao, "A multiperiod set covering location model for dynamic redeployment of ambulances," Computers and Operations Research, vol. 35, no. 3, pp. 814-826, 2008.

[3] W. Yi and L. Özdamar, "A dynamic logistics coordination model for evacuation and support in disaster response activities," European Journal of Operational Research, vol. 179, no. 3, pp. 1177-1193, 2007.

[4] M. S. Chang, Y. L. Tseng, and J. W. Chen, "A scenario planning approach for the flood emergency logistics preparation problem under uncertainty," Transportation Research E, vol. 43, no. 6, pp. 737-754, 2007.

[5] C. G. Rawls and M. A. Turnquist, "Pre-positioning of emergency supplies for disaster response," Transportation Research $B$, vol. 44, no. 4, pp. 521-534, 2010.

[6] H. O. Mete and Z. B. Zabinsky, "Stochastic optimization of medical supply location and distribution in disaster management," International Journal of Production Economics, vol. 126, no. 1, pp. 76-84, 2010.

[7] W. Wang, F. Hou, H. Tan, and H. Bubb, "A framework for function allocations in intelligent driver interface design for comfort and safety," International Journal of Computational Intelligence Systems, vol. 3, no. 5, pp. 531-541, 2010.

[8] W. Wang, Y. Mao, J. Jin et al., "Driver's various information process and multi-ruled decision-making mechanism: a fundamental of intelligent driving shaping model," International Journal of Computational Intelligence Systems, vol. 4, no. 3, pp. 297-305, 2011. 


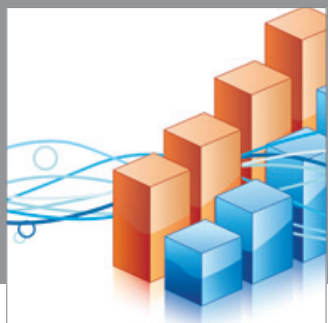

Advances in

Operations Research

mansans

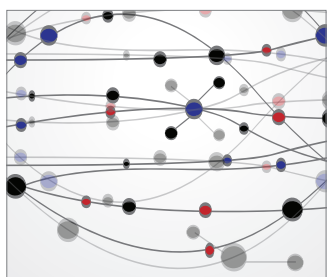

The Scientific World Journal
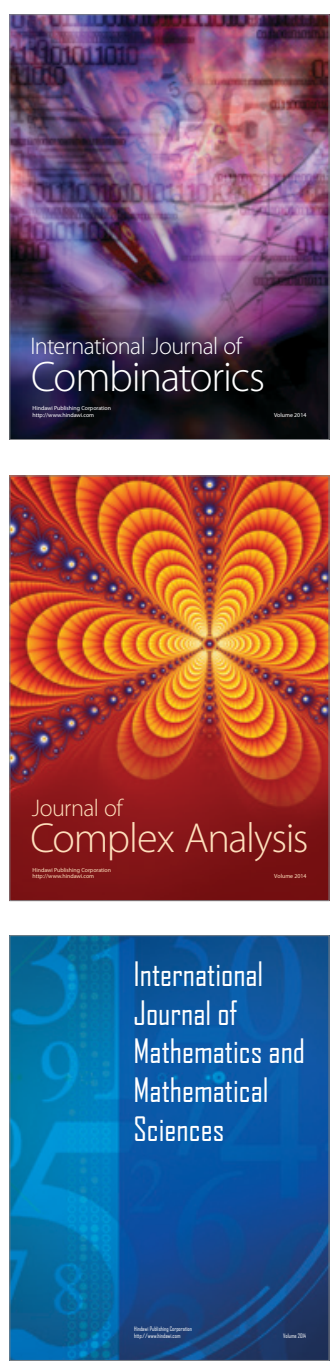
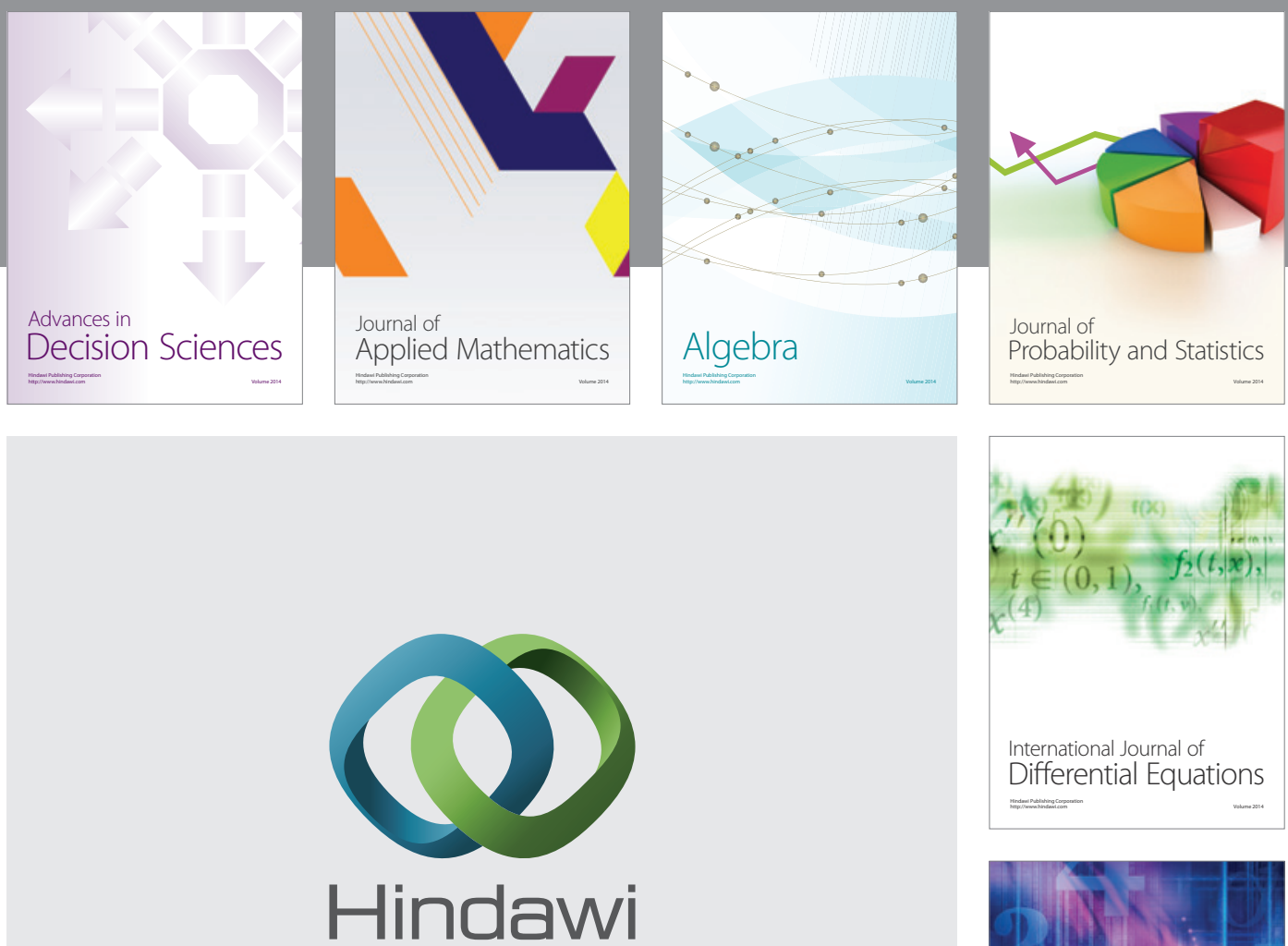

Submit your manuscripts at http://www.hindawi.com
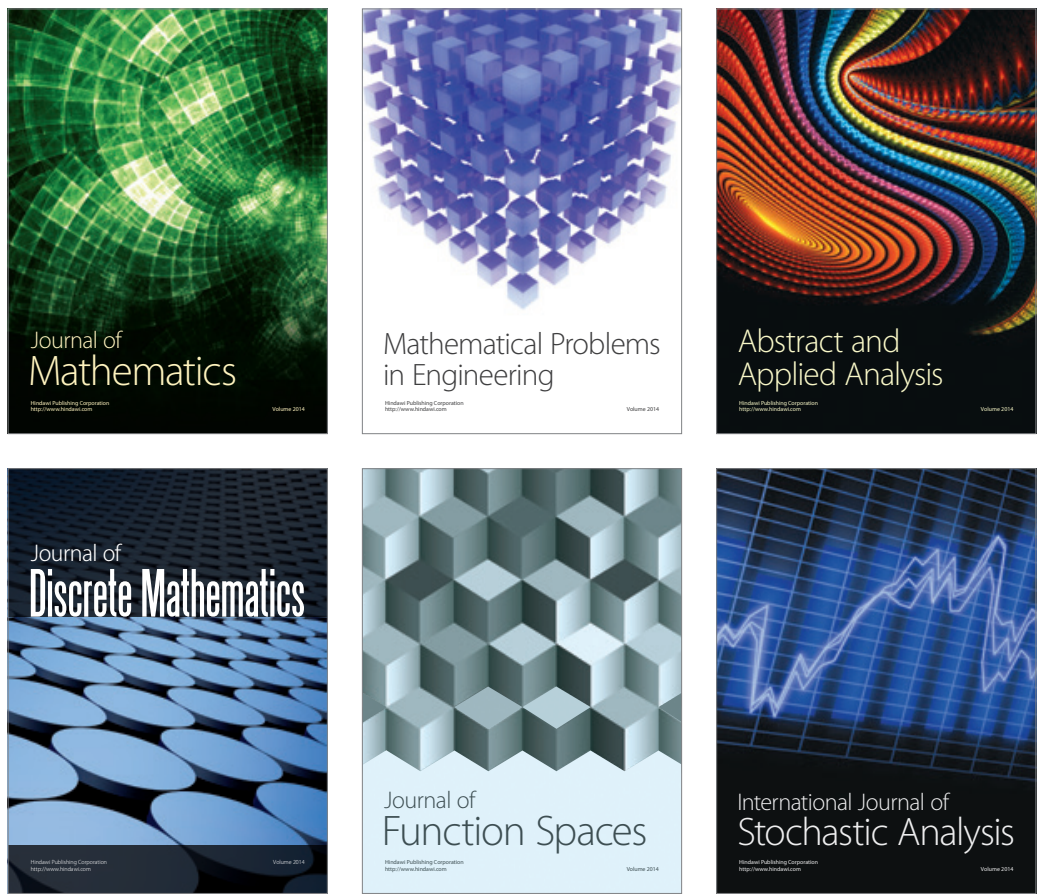

Journal of

Function Spaces

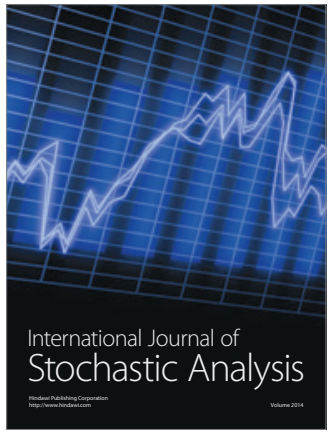

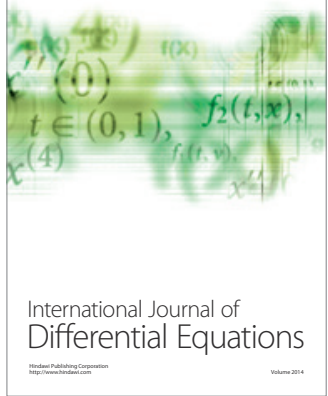
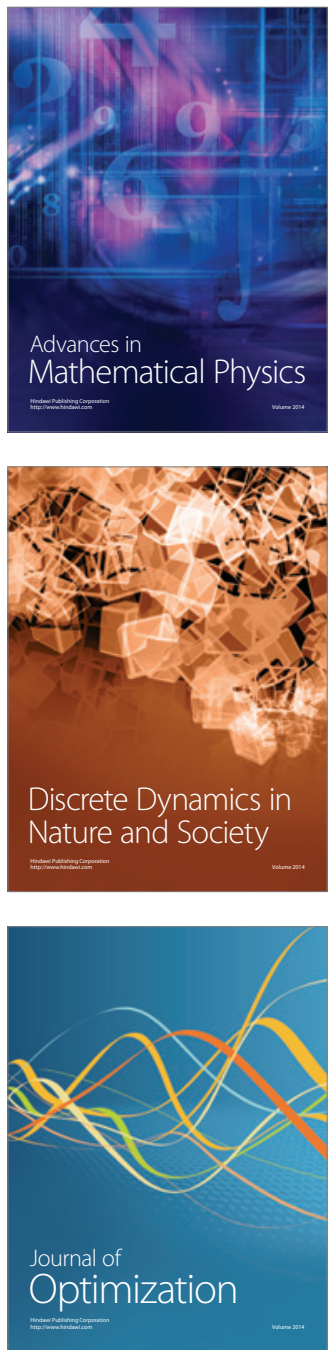